\title{
THE PARTICLE SEARCH IN A QUANTUM FIELD MODEL
}

\author{
BY JAMES GLIMM ${ }^{1}$ AND ARTHUR JAFFE ${ }^{2}$ \\ Communicated by Murray Protter, February 6, 1973
}

The goal of quantum field theory is a description of elementary particles. When successful, this description should be both a mathematical theory and a law of nature. In our approach, we emphasize the construction of specific field theory models. The first motive for this emphasis is the mathematical consistency of quantum field theory. In addition, we follow closely the formal ideas of physics in order to provide a more solid foundation for the main task of quantum field theory: the study of elementary particles.

It is clear by now that the first motive has been attained in two spacetime dimensions. In two space-time dimensions, quantum fields with nonlinear interactions have been constructed $[1],[2]$. For the polynomial boson $\left(\mathscr{P}(\phi)_{2}\right)$ interactions with small coupling constant, all the Wightman axioms have been verified [5].

We announce two new results. The first is an estimate which is a major technical step toward establishing the above results in three space-time dimensions. The second result concerns the physical interpretation of our two dimensional models (the second motive of our study). In $\mathscr{P}(\phi)_{2}$ models with a small coupling constant, we establish the existence of particles and we verify the Haag-Ruelle axioms for scattering. Thus we conclude that the $\mathscr{P}(\phi)_{2}$ model has the qualitative structure required for the description of (many particle) scattering experiments.

In our first result we consider the $\phi_{3}^{4}$ interaction: a $\phi^{4}$ coupling in the interaction Hamiltonian for boson fields in three space-time dimensions. We introduce a space cut-off $g \in C_{0}^{\infty}\left(R^{2}\right), 0 \leqq g \leqq 1$, in the interaction Hamiltonian density, and we let the resulting total Hamiltonian (with infinite counterterms from second and third order perturbation theory) be denoted $H(g)$. Let $A(g)$ denote the set of points within distance 1 of supp $g$.

THEOREM 1. With a constant $c$ independent of $g,-c A(g) \leqq H(g)$.

In our second result, we consider the $\mathscr{P}(\phi)_{2}$ theory with the dimensionless bare coupling constant $\lambda / m_{0}^{2}$ small. Let $P_{0}$ and $P_{1}$ denote the energy and momentum operators. The mass operator is $M=\left(P_{0}^{2}-P_{1}^{2}\right)^{1 / 2}$.

AMS (MOS) subject classifications (1970). Primary 81A18; Secondary 81 A48.

${ }^{1}$ Supported in part by the National Science Foundation, NSF-GP-24003.

${ }^{2}$ Supported in part by the Air Force Office of Scientific Research, Contract AF 4462070-C0030 and the National Science Foundation Grant GP-31239X. 
Zero is an eigenvalue of $M, P_{0}$ and $P_{1}$. For $\lambda / m_{0}^{2}$ small there is a gap above zero in the spectrum of $M[5]$, namely $m=\inf \{\sigma(M) \sim 0\}>0$. Our second result establishes a gap above $m$ in the spectrum of $M$.

THEOREM 2. In the $\mathscr{P}(\phi)_{2}$ model with $\lambda / m_{0}^{2}$ small, $m$ is an isolated eigenvalue for $M$. The corresponding eigenspace is Poincaré invariant (because $M$ is) and on this subspace the Poincaré group acts as an irreducible mass $m$ representation.

By the Haag-Ruelle scattering theory [6], an isometric $S$ matrix exists, together with asymptotic in and out fields. The in and out fields label the particles in the scattering states.

The proof of these theorems will appear elsewhere [3], [4].

\section{BIBLIOGRAPHY}

1. J. Glimm and A. Jaffe, Quantum field theory models, Statistical Mechanics and Quantum Field Theory (edited by C. de Witt and R. Stora), Gordon and Breach, New York, 1971.

2. - Boson quantum field models, Mathematics of Contemporary Physics (edited by R. Streater), Academic Press, New York.

3. - Positivity of the $\phi_{3}^{4}$ Hamiltonian, Fortschr. Physik (to appear).

4. - The particle structure in the $P(\phi)_{2}$ model (preprint).

5. J. Glimm and T. Spencer, The Wightman axioms and the mass gap for the $P(\phi)_{2}$ quantum field theory (preprint).

6. K. Hepp, On the connection between Wightman and LSZ quantum field theory, Axiomatic Field Theory (edited by M. Chretien and S. Deser), Gordon and Breach, New York, 1966.

Courant Institute of Mathematical Sciences, New York University, New York, NEW YORK 10012

Harvard University, CAMbridge, MassachusetTs 02138 